\title{
De Volta para o Futuro - Parte II: Stents Auto-expansíveis?
}

\author{
Ver artigo relacionado \\ na página 341
}

\author{
Hiram G. Bezerra ${ }^{1}$, Marco A. Costa ${ }^{1}$
}

0 Wallstent, um stent auto-expansível (AE), foi a primeira prótese intracoronária utilizada na tentativa de reduzir a oclusão aguda e a reestenose ${ }^{1}$. Logo se observou, porém, que o comprimento e o diâmetro das próteses tinham correlação direta com o porcentual de reestenose, e que a liberação precisa era um importante fator nos índices de reestenose ${ }^{2}$. O Wallstent teve então a difícil tarefa de competir com as próteses expansíveis por balão (stents balão-expansíveis - BE), que, pelo mais fácil manuseio, pela maior precisão de liberação e pelas taxas semelhantes ou meIhores de reestenose, logo se impuseram como preferência do cardiologista intervencionista e transformaram, de forma definitiva, a revascularização coronária ${ }^{3}$. A primeira experiência com os stents BE foi realizada no Brasil, sob direção do prof. J. Eduardo Sousa, no Instituto Dante Pazzanese de Cardiologia - o mesmo grupo que hoje apresenta os dados de uma nova prótese AE para tratamento de vasos de fino calibre.

Apesar da relativa curta sobrevida no leito coronário, as próteses $\mathrm{AE}$ gozam de grande sucesso em outros leitos vasculares. Talvez esse sucesso tenha sido a razão para a insistência do uso de stent $\mathrm{AE}$ no âmbito da Cardiologia Intervencionista. Nova tentativa de uso desses stents aconteceu com os estudos Symbiots, que utilizou stents revestidos com politetrafluoretileno (PTFE) para o tratamento de pontes de safena ${ }^{4}$. Esse parecia ser o caminho natural, pois tratava-se de vasos de maior calibre e lesões mais longas, condição que de certa forma mimetiza a intervenção em leitos vasculares periféricos. No entanto, mesmo nessas condições teoricamente ideais para a utilização de stent $A E$, essas próteses cursaram com maiores taxas de reestenose, quando comparadas aos stents $\mathrm{BE}$, mas $\mathrm{O}$ fator desapontador preponderante foi o fato de não prevenirem fenômenos embólicos ${ }^{5}$. Parecia então que $\mathrm{O}$ assunto stent $\mathrm{AE}$ para o cardiologista intervencionista focado no tratamento coronário havia chegado ao fim, ficando sua utilização restrita a situações raras, casos esparsos de tratamento de aneurismas ou na cardiopatia congênita.
De maneira muito inovadora, o tema stent $\mathrm{AE}$ ressurge no artigo apresentado por Chamié et al. ${ }^{6} \mathrm{em}$ uma subpopulação de portadores de doença coronária quase que antagônica aos trabalhos anteriores, no difícil cenário da intervenção em vasos de pequeno calibre com lesões curtas. A intervenção em vasos pequenos é desafiadora por inúmeras razões, já que as atuais próteses não foram desenhadas, mas adaptadas, para lidar com tais situações anatômicas. As limitações associadas ao desenho das atuais próteses influenciam tanto os resultados primários como tardios. No que diz respeito ao resultado primário, pode-se ressaltar a dificuldade de navegabilidade em leitos coronários distais e a falta de flexibilidade para se adaptar à natureza tortuosa dos vasos de fino calibre. Após vencido o problema de navegabilidade, o cardiologista intervencionista ainda tem que lidar com as dissecções de bordas, mais freqüentes nessas situações. Em relação aos resultados tardios, a reestenose em vasos pequenos ainda é um problema, mesmo na era dos stents farmacológicos, sendo a reestenose de borda mais comum nessa subpopulação ${ }^{7}$. Stents pouco flexíveis podem resultar em grande estresse de parede nas bordas dos vasos pequenos, gerando, por conseguinte, hiperplasia neo-intimal. Os relatos de fratura de stent também podem ser explicados pela falta de conformidade das atuais próteses em segmentos tortuosos, situação comum em vasos distais e de fino calibre. Testou-se, em vários estudos, o tratamento com angioplastia com balão sem implante do stent, o que parece não ser a solução ideal, visto que o intervencionista não pode mais aceitar a imprevisibilidade dos resultados agudos da angioplastia sem stent, além da falta de benefício dessa estratégia nos resultados tardios.

O stent AE apresentado nesse estudo possui uma característica única: o fato de ter o menor perfil de prótese endovascular já construído (0,014 polegada), semelhante ao fio-guia utilizado para a liberação de stents convencionais. Esse sistema permite ainda o uso de balão sobre o mesmo guia para pré- e pósdilatação. $\mathrm{O}$ acesso aos leitos mais distais e tortuosos fica facilitado por se tratar de stent AE, mais flexível,

\footnotetext{
1 University Hospitals Harrington-McLaughlin Heart \& Vascular Institute - Case Western Reserve University - Cleveland, Estados Unidos. Correspondência: Marco A. Costa. 11100 Euclid Avenue - Lakeside 3001 - Cleveland, OH, USA - 44106-5038

E-mail: marco.costa@uhhospitals.org

Recebido em: 18/8/2008 • Aceito em: 21/8/2008
} 
e pela capacidade de se conformar à tortuosidade dos vasos pequenos, muito superior à dos stents BE. Além disso, esse stent pode ser introduzido através do lúmen de um balão de angioplastia convencional ou de microcateteres com lúmen $\geq 0.014$ polegada. Nesse estudo, não foram demonstradas diferenças quanto ao volume luminal nas bordas quando comparado a stents $\mathrm{BE}$, dedicados a vasos pequenos. Acreditamos que essa instigante questão ainda deverá ser testada em séries maiores e em pacientes com anatomia mais desafiadora. Apesar de ser atraente o fato de a contínua expansão do stent poder resultar em maior manutenção do lúmen, como demonstrado nessa pequena série, ainda pouco se sabe sobre a biologia vascular implicada nesse mecanismo. Se, por um lado, a injúria primária do implante pode ser minimizada com o stent $A E$, por outro lado o contínuo estímulo na parede vascular provocado por esse stent pode resultar em inflamação crônica ${ }^{8,9}$. Não acreditamos que a questão da reestenose intra-stent seja o ponto de maior interesse nessa prótese, pois na era dos stents eluídos por medicação o stent CardioMind ${ }^{\circledR}$ também seguirá nessa direção. Atualmente, o estudo CARE II está sendo conduzido com o mesmo sistema CardioMind ${ }^{\circledR}$, porém associado a um polímero biodegradável. Aquilo que torna esse stent único é seu potencial em navegabilidade e conformidade, o que amplia de forma quase ilimitada o acesso ao leito coronário.

Do ponto de vista clínico, entretanto, devemos sempre ter em perspectiva um balanço positivo entre risco e benefício ao tratar vasos de fino calibre. Uma tecnologia que não proporcione benefícios clínicos, por mais inovadora e atraente que seja, estará fadada ao fracasso. O sistema CardioMind ${ }^{\otimes}$ tem o potencial de minimizar os riscos agudos do procedimento ou até mesmo tornar possível uma intervenção que outrora seria impossível. Mas o benefício clínico da intervenção em vasos pequenos tem que ser avaliado dentro de perspectiva clínica. Estenoses em vasos de fino calibre raramente causam distúrbios hemodinâmicos ou isquemia significativa, como demonstrado previamente em um estudo também iniciado no Instituto Dante Pazzanese de Cardiologia (PHANTOM), que utilizou e monitorou a reserva de fluxo fracionada do miocárdio em pacientes com vasos $<2,8 \mathrm{~mm}$ de diâmetro ${ }^{10}$. Por outro lado, na prática diária do cardiologista intervencionista, não raro nos deparamos com pacientes que apresentam sintomas isquêmicos de difícil controle e anatomia tortuosa em vaso de fino calibre. Felizmente parece que em futuro não muito distante teremos uma tecnologia que vai oferecer uma solução de revascularização minimamente invasiva e de baixo risco.

Temos acompanhado o desempenho desse stent inovador com grande interesse, um exemplo de tecno- logia revisitada com grande potencial de sucesso. Assim como em editorial prévio relativo ao trabalho envolvendo cateter-balão eluído em paclitaxel ${ }^{11}$, o artigo original apresentado nesta edição da Revista Brasileira de Cardiologia Invasiva (RBCI) também representa "uma volta ao futuro". É mais um exemplo do dinamismo da Cardiologia Intervencionista e da importância de se olhar para o passado com a perspectiva de futuro.

\section{REFERÊNCIAS BIBLIOGRÁFICAS}

1. Sigwart U, Puel J, Mirkovitch V, Joffre F, Kappenberger L. Intravascular stents to prevent occlusion and restenosis after transluminal angioplasty. N Engl J Med. 1987;316(12):701-6.

2. Foley DP, Pieper M, Wijns W, Suryapranata H, Grollier G, Legrand $V$, et al. The influence of stent length on clinical and angiographic outcome in patients undergoing elective stenting for native coronary artery lesions; final results of the Magic 5L Study. Eur Heart J. 2001;22(17):1585-93.

3. Nageh T, de Belder AJ, Thomas MR, Williams IL, Wainwright RJ. A randomised trial of endoluminal reconstruction comparing the NIR stent and the Wallstent in angioplasty of long segment coronary disease: results of the RENEWAL Study. Am Heart J. 2001;141(6):971-6.

4. Laarman GJ, Kiemeneij F, Mueller R, Guagliumi G, Cobaugh $M$, Serruys PW. Feasibility, safety, and preliminary efficacy of a novel ePTFE-covered self-expanding stent in saphenous vein graft lesions: the Symbiot II trial. Catheter Cardiovasc Interv. 2005;64(3):361-8.

5. Blackman DJ, Choudhury RP, Banning AP, Channon KM. Failure of the Symbiot PTFE-covered stent to reduce distal embolization during percutaneous coronary intervention in saphenous vein grafts. J Invasive Cardiol. 2005;17(11):609-12.

6. Chamié D, Costa Jr JR, Abizaid A, Feres F, Devito F, Mattos LA, et al. Stents auto-expansíveis versus balão-expansíveis para tratamento de lesões em vasos de fino calibre: estudo com ultra-som intracoronário tridimensional. Rev Bras Cardiol Invas. 2008;16(3):341-52.

7. Togni M, Eber S, Widmer J, Billinger M, Wenaweser $P$, Cook S, et al. Impact of vessel size on outcome after implantation of sirolimus-eluting and paclitaxel-eluting stents: a subgroup analysis of the SIRTAX trial. J Am Coll Cardiol. 2007;50(12):1123-31.

8. König A, Klauss V, Regar E, Rieber J, Casella G, Theisen K, et al. Serial intravascular ultrasound and quantitative coronary angiography after self-expandable wallstent coronary artery implantation. Am J Cardiol. 2000;86(9):1015-8, A10.

9. Kobayashi Y, Honda Y, Christie GL, Teirstein PS, Bailey SR, Brown $\mathrm{CL} 3^{\text {rd }}$, et al. Long-term vessel response to a selfexpanding coronary stent: a serial volumetric intravascular ultrasound analysis from the ASSURE Trial. A Stent vs. Stent Ultrasound Remodeling Evaluation. J Am Coll Cardiol. $2001 ; 37(5): 1329-34$.

10. Costa MA, Sabate M, Staico R, Alfonso F, Seixas AC, Albertal $M$, et al. Anatomical and physiologic assessments in patients with small coronary artery disease: final results of the Physiologic and Anatomical Evaluation Prior to and After Stent Implantation in Small Coronary Vessels (PHANTOM) trial. Am Heart J. 2007;153(2):296.e1-7.

11. Camenzind E. Treatment of in-stent restenosis: back to the future? N Engl J Med. 2006;355(20):2149-51. 